\title{
Primary pericardial mesothelioma presenting as pericardial constriction: a case report
}

\author{
S Suman, P Schofield, S Large
}

Heart 2004;90:e4 (http://www.archdischild.com/cgi/content/full/90/1/e4)

A 19 year old man presented with a six month history of chest pain, dyspnoea, and lethargy and was found on an echocardiogram to have a dilated left ventricle with a small pericardial effusion. Ramipril and a course of steroids were tried but serial echocardiograms showed a persistently thickened pericardium and slowly developing features of constriction. On computed tomography, a large mediastinal mass encasing the heart, along with para-aortic and paratracheal lymphadenopathy, was found. Right heart catheter studies showed equal pressures in all four chambers. His deteriorating clinical condition led to a pericardiectomy. Histology confirmed primary pericardial mesothelioma. The patient died soon after surgery.

$\mathrm{P}$ ericardial mesotheliomas are extremely rare and have an overall poor prognosis. Common clinical features are constrictive pericarditis, cardiac tamponade, and cardiac failure. Surgery can be curative in localised cases. Response to radiotherapy is poor. Pericardiectomy to alleviate symptoms and chemotherapy to reduce tumour mass are commonly used palliative measures.

\section{CASE REPORT}

A 19 year old male student presented with a six month history of chest pain, lethargy, and occasional dyspnoea. His medical history was unremarkable. He was first admitted to his local hospital in June 2002 after chest radiography undertaken by his general practitioner had shown cardiomegaly. ECG on admission showed widespread $\mathrm{T}$ wave inversion in the inferior and lateral leads. Serial cardiac enzymes were normal. Viral and antistreptolysin O titres were negative but his inflammatory markers were raised (C reactive protein 76 and erythrocyte sedimentation rate 106). A transthoracic echocardiogram showed a dilated left ventricle with impaired function and a small pericardial effusion. Pericarditis was provisionally diagnosed and treatment with ramipril was started.

Serial outpatient echocardiograms showed a thickened pericardium and a persistent pericardial effusion. Prednisolone was added to his treatment and although this resulted in some resolution of the effusion, along with improvement in the patient's general condition and inflammatory markers, the subsequent echocardiogram showed patterns strongly indicative of pericardial constriction. In view of the protracted course of his illness a rheumatologist's opinion was sought. After a series of assays and tests, an autoimmune cause was ruled out.

The patient was weaned from prednisolone but this unfortunately resulted in his clinical deterioration and readmission within a few weeks with signs of pericardial constriction (September 2002). An echocardiogram now showed severe biventricular impairment and diuretics were started. A large mediastinal mass encasing the heart and paratracheal lymphadenopathy were found on computed tomography (CT) during this admission (fig 1). In addition there was evidence of a right pleural effusion and para-aortic lymphadenopathy, which raised the possibility of an underlying lymphoma (fig 2).

The patient was referred to the regional cardiology centre at Papworth for further assessment and right and left heart catheter studies with a view to obtaining a biopsy and performing a pericardiectomy. Coronary angiography showed unobstructed coronary arteries and angiography showed moderate impairment of both ventricles. Pressures were matched in all four chambers. The wave form of pressure measurements in diastole followed a dip and plateau pattern suggestive of pericardial constriction. A CT guided pericardial aspiration was also attempted but no fluid could be aspirated and the material seemed gelatinous.

The patient developed a deep vein thrombosis in the right subclavian and internal jugular veins in November 2002. This unusual site of venous thrombosis in the absence of previous instrumentation strongly suggested an underlying malignancy. His general condition deteriorated and pericardiectomy was undertaken.

At surgery, the pericardium was $2 \mathrm{~cm}$ thick, white, homogeneous, and very strongly adherent to the myocardium. As a result, resection of the pericardium was incomplete. Histological analysis showed a primary pericardial mesothelioma of the epithelial type (fig 3). Postoperatively the patient's condition deteriorated rather rapidly with a low cardiac output state and he soon died. At the postmortem examination, the pericardium was thickened, encased the entire heart and extended up to the aorta and pulmonary arteries. There was no evidence of asbestos fibres in the lungs and the only other abnormalities were in keeping with congestive cardiac failure.

\section{DISCUSSION}

Primary pericardial tumours are rare and can be benign (teratoma, fibroma, angioma, lipoma) or malignant (mesothelioma, sarcoma). Secondary tumours are more common, metastasising mostly from the lung, breast, melanomas, lymphoma, or leukaemia. ${ }^{1}$ The incidence of malignant pericardial involvement has been reported in the literature from $0.15-21 \%$ of all patients with an underlying malignancy. It is estimated that of all patients with malignant cardiac involvement, about $85 \%$ have pericardial involvement. Prognosis is poor with survival after diagnosis ranging from six weeks to 15 months. ${ }^{1}$

Pericardial mesothelioma is extremely rare, although it is the most common primary malignant pericardial tumour. Its incidence was $<0.0022 \%$ among 500000 cases in a large necropsy study. ${ }^{2}$ Approximately 200 cases have been reported so far and only $25 \%$ of these were antemortem diagnoses. Pericardial mesothelioma can present as a localised or as a diffuse mass. Three histological types have been described: epithelial, spindle cell, and mixed. ${ }^{3}$ There is a possible 


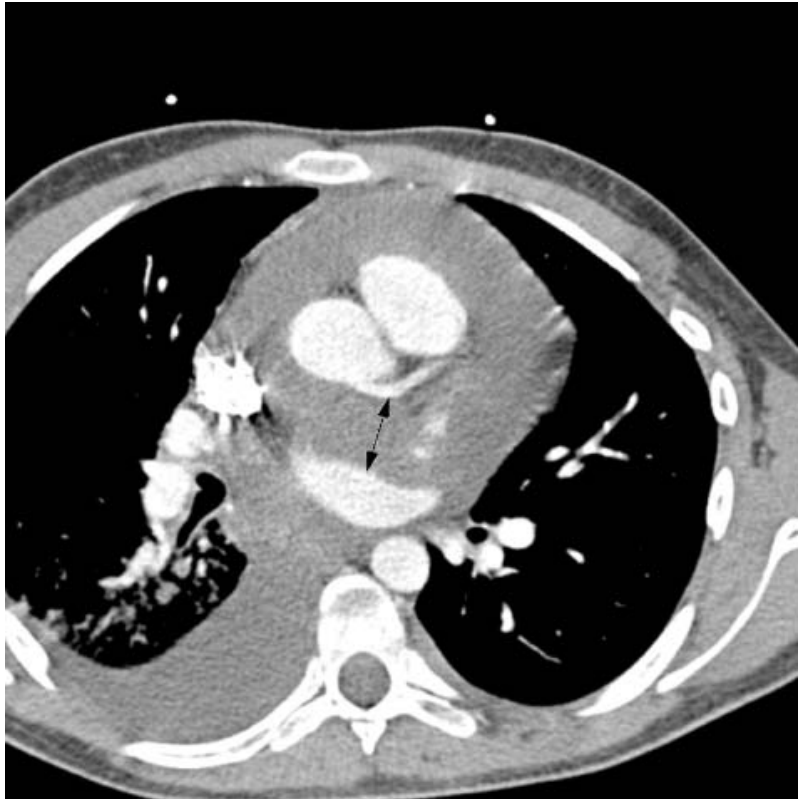

Figure $1 \mathrm{CT}$ section through the aortic root showing large right pleural effusion and a low attenuation soft ring encasing the heart. The posterior displacement of the left atrium from the main coronary artery and LAD suggests this is due to soft tissue rather than fluid (arrows).

relation to asbestos exposure but this is seen only in cases with coexistent asbestos related pleural disease. ${ }^{4}$

The onset of symptoms is usually insidious. Common clinical manifestations of pericardial mesothelioma are constrictive pericarditis, pericardial effusion, cardiac tamponade, and heart failure caused by myocardial infiltration. Further symptoms may arise due to compression of coronary arteries and local spread into the surrounding great vessels. Primary mesothelioma can also mimic tuberculous pericarditis or intra-atrial myxomas. Distant metastasis, conduction

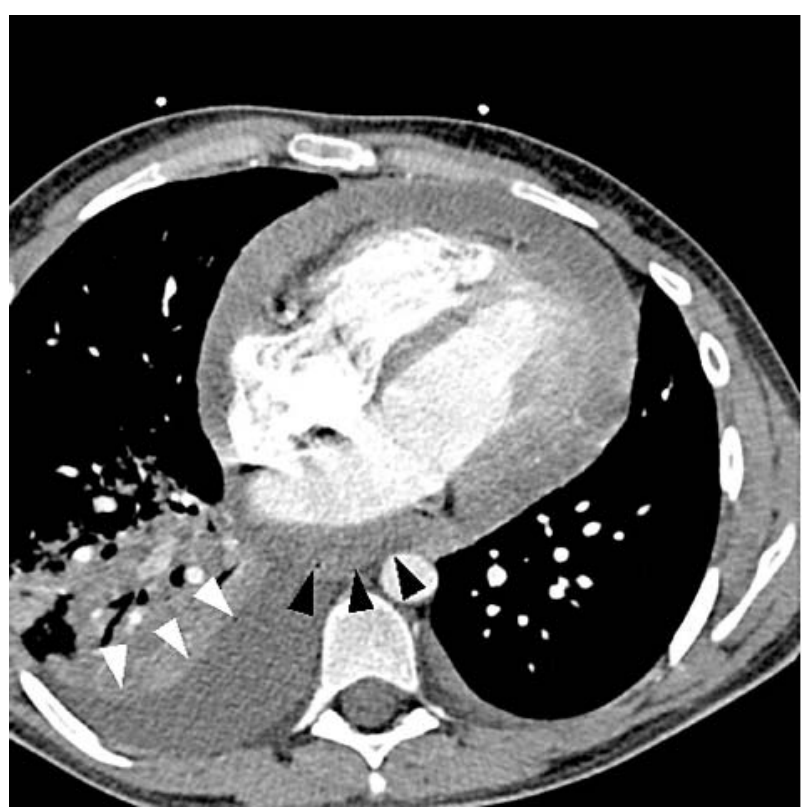

Figure 2 CT section more inferiorly showing slight difference in attenuation between pleural fluid (white arrows) and pericardial mesothelioma (black arrows). Cardiac silhoutte is enlarged, although the heart is small.

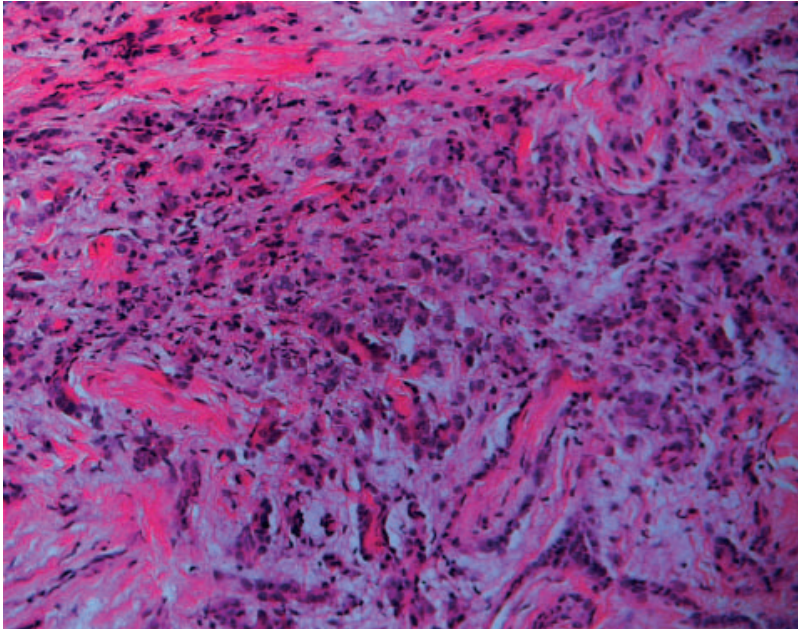

Figure 3 Histology: section of pericardium showing diffuse infiltration by epithelioid cells which had an immunohistochemical profile in keeping with malignant mesothelioma.

block due to myocardial infiltration, ${ }^{5}$ and tumour embolism causing neurological deficits ${ }^{6}$ have also been reported.

Pericardial fluid in pericardial mesothelioma can be difficult to aspirate. Echocardiography is the most commonly used initial investigative tool. Magnetic resonance imaging and CT are useful in showing the extent of involvement of contiguous structures and the degree of constriction. Other investigations such as immunohistochemistry, cytological examination, and high pericardial hyaluronic acid content of the pericardial aspirate can be diagnostic. The abnormal pericardium has also been known to take up gallium 67 extensively at scintigraphy. ${ }^{7}$

Pericardial mesothelioma responds poorly to radiotherapy. Cyclical combination chemotherapy with doxorubicin, vincristine, and cyclophosphamide may reduce the tumour mass. ${ }^{7}$ Surgical resection can be curative in localised cases. Palliative treatment includes pericardiectomy, which prevents cardiac tamponade and relieves constriction. Alternatively, a pericardial window can be formed to introduce chemotherapeutic agents. ${ }^{8}$ Areas of ongoing research include intracavitary chemotherapy and irradiation. Photodynamic treatment, inhibition of growth factors, vaccines, and adenoviral molecular chemotherapy are also being explored. ${ }^{9}$

\section{ACKNOWLEDGEMENTS}

Thanks to Dr Martin Goddard, Consultant Histopathologist, Papworth Hospital and Dr Richard Coulden, Consultant Radiologist, Papworth Hospital for the slides.

\section{Authors' affiliations}

S Suman, P Schofield, S Large, Departments of Cardiology and Cardiac Surgery, Papworth Hospital, Papworth, Cambridge, UK

Correspondence to: Dr Shivani Suman, Department of Cardiology, Papworth Hospital, Papworth, Cambridge, UK;

shivani.suman@virgin.net

Accepted 11 July 2003

\section{REFERENCES}

1 Kralstein J, Frishman W. Malignant pericardial diseases: diagnosis and treatment. Am Heart J 1987; 113:785-90.

2 Gossinger HD, Siostrznek P, Zangeneh $M$, et al. Magnetic resonance imaging findings in a patient with pericardial mesothelioma. Am Heart $J$ 1988; 115:1321-2.

3 Yilling FP, Schlant RC, Hertzler GL, et al. Pericardial mesothelioma. Chest 1982:81:520-3. 
4 Loire $\mathbf{R}$, Tabib A. [Malignant mesothelioma of the pericardium: an anatomoclinical study of 10 cases]. Arch Mal Coeur Vaiss 1994;87:255-62.

5 Fazekas T, Tiszlavicz L, Ungi I. [Primary malignant pericardial mesothelioma] Orv Hetil 1991; 132:2677-80.

6 Szczechowski L, Janiec K. [Pericardial mesothelioma as a very rare cause of recurrent cerebral emboli]. Wiad Lek 1992;45:857-61.
7 Nambiar CA, Tareif HE, Kishore KU, et al. Primary pericardial mesothelioma: one year event free survival. Am Heart J 1992;124:802-3.

8 Piwowarska W, Nessler B, Pietrzak I, et al. [Diagnostic difficulties in a 32 year old patient with cardiac tamponade]. Kardiol Pol 1993;38:209-12.

9 Eren NT, Akar AR. Primary pericardial mesothelioma. Curr Treat Options Oncol 2002;3:369-73. 